\title{
Matter and information as attributes of substance
}

\author{
R.E. Zimmermann ${ }^{1,2,3, a}$ \\ 1 Faculty Studium Generale, Munich University of Applied Sciences, Munich, Germany \\ 2 Clare Hall, Cambridge, UK \\ 3 Institute for Design Science e.V., Munich, Germany
}

Received 19 November 2016 / Received in final form 19 November 2016 Published online 10 February 2017

\begin{abstract}
Until now, there is no explicit clarity about the question whether information, as similar to energy and mass, can be visualized as fundamental constituent of this universe or not, a question which is presently disputed. We follow here the line of argument which follows the first alternative, and give reasons for this by starting in terms of a metaphysical conception.
\end{abstract}

\section{The metaphysical perspective}

We follow here the traditional line of argument that originates (within the framework of sufficiently modern thought) in the substance metaphysics of Spinoza. The basic idea is to notice that the world is not as we observe it, simply due to the restrictions which determine the human cognitive capacity. The fundamental categories by which humans model the (for them) observable world such as space, time, matter and so forth, are the results of a long process of human reflexion rather than properties that would be really available in the world and exist even when no human beings would be around. We thus take what is called the "realistic" position (although it would be more precise to call it "actualistic" instead), meaning that we believe there is a world which is constituted independently of human beings. But we also believe that human beings can never understand the whole truth of worldly existence in the sense that we can never completely realize how the world really is. We can only see how the world is actually for us. (The word "us" includes all possible humans that live on other planets in the universe having in common their biological constitution in terms of carbon and water.) To utilize a concept of Spinoza's: We observe modality, but not reality proper.

If so, we introduce a hierarchy of forms of existence (types of being) such that everything which is according to our observation (in the terminology of Heidegger's this is an ontic concept) must have been possible beforehand. Hence, what we usually call existence is the permanent transition of possibility into actuality. In other words: There is a field of possibilities out of which the actual (i.e. modality) is emerging. Because the universe is not necessarily ergodic (i.e. not all available possibilities must be actualized eventually), the number of available possibilities is considerably larger than the number of actualized possibilities. So in order to produce actual objects

\footnotetext{
${ }^{a}$ e-mail: rainer.zimmermann@hm.edu
} 
in the observable world, emergence takes place such that from the beginning on, there is a choice. There is always a choice, if the number of available possibilities is larger than the number of actualized possibilities. The spontaneous emergence of configurations of forms (structures) represents thus a selection from a number of possible configurations. Hence, because there is this obvious discrepancy between what there could be and what there actually is, we can call this a first difference. It is in this sense that we can utilize the formulation of Bateson's: Information is the difference which makes a difference. If in particular, we discuss the initial emergence which is the emergence of the universe altogether, considering the latter as a maximal system then, we can conclude that information must be present from the beginning on because initial emergence is grounded in a first choice.

\section{The physical perspective}

Note however that we have defined physical quantities as something which is epistemologically intrinsic to the world we observe. Hence, on the level of the field of possibilities, they cannot be identical with what we have introduced within the framework of physical theories. In the following we call energy-mass in physical terms (referring to their equivalence but qualitative difference) matter for simplicity (and in order to find the connection to philosophical tradition). While the dual pair entropy-structure will be called information for short. These dual pairs have been discussed recently in more detail elsewhere [1]. As far as we can treat these quantities in physical terms (which also includes the performance of experiments in the laboratory), they are part of our observable world. But they are not part of the field of possibilities in the first place. In other words: As seen under the perspective of the latter, they are modal projections rather of what there really is. But for the purpose of technical applications, this conceptual viewpoint suffices (for daily use, so to speak), and the physicist asks whether it is functioning after all. The philosopher however, has to ask for the conceptual foundations, also. And because we aim here at an approach which shall produce definitions of concepts, we have to take the position of the philosopher so that in the end, the ontological difference is not trivial at all.

We notice now: Both energy-mass (matter) and entropy-structure (information), respectively, must be present from the beginning on. The one is providing the capacity to let something emerge at all, the other is providing the rules that tell what might actually emerge and how. It does not matter here that what we call matter and information might turn out to be mere projections of something which is much more fundamental and cannot be observed and conceptualized properly by us in principle, because we simply reflect about these concepts according to our cognitive capacity and as far as it goes. As to the sceptical part of this reflexion it is sufficient then that applications work in the end, but as to the speculative part of this reflexion we might even gain some new heuristic insight which is a kind of excess capacity [2].

The philosophical tradition tells us that we can call the ground of our observable world substance. Hence, matter and information turn out to be those two attributes by which we can gain access to substance. Note that the transition from the nonobservable world to the observable world (from the possible into the actual) secures the continuity of conceptual functions: It is loops in the sense of quantum gravity that by their energy as well as entropy processing fulfil the definition of agents as given by Stuart Kauffman some time ago. On the most fundamental level of physics (at the Planck scale), six loops actually co-operate in order to form a hexagonal segment of the underlying spin network. It is this spin network which defines the level where space and time themselves are quantized. The hexagonal pattern is not at all arbitrary 
here, because its formal structure secures the relevance of the famous transformation group SL $(2, \mathrm{C})$. On the one hand, this guarantees the well-known relationships of relativistic and quantum physics, on the other, this demonstrates the isomorphism of the spin network with what we can call a quantum computer. In other words, already on the most fundamental level of physics does the universe as maximal system show up in terms of information, though this is not classical information as we know it instead, it is quantum information.

This result remains invariant when looking for higher levels of evolution: going from the Planck scale upward to the Compton scale, to the Schwarzschild scale and further up to chemical, biological and other structures. Somewhere in between the Compton scale and the Schwarzschild scale, the transition to classicity takes place due to the order of magnitudes encountered. A unified methodology which is able to grasp all the important aspects of this overall view in terms of substantial attributes called matter and information, respectively, has been recently provided by category theory, or more precisely: by a variant of it called topos theory. I have discussed this point in detail within the works quoted so far.

\section{Outlook: Differentiating the concepts}

Recently, Carlo Rovelli has introduced an argument which is very much in favour of what we have done here already [3]. Although he comes very quickly to living structures within the observable world, the generalized concept of "relative information" appears to be very helpful for physical systems after all: The idea is that if we have two systems $A$ and $B$, respectively, that can be in states $N_{A}$ and $N_{B}$, then without any constraints, the interactive complex $(\mathrm{A}, \mathrm{B})$ can be in states $\mathrm{N}_{A} \times \mathrm{N}_{B}$. However, practically all possible systems are subject to constraints. Hence, the real bundle of possible states is described by some $\mathrm{N}_{A B}$ such that this is strictly smaller than $\mathrm{N}_{A} \times \mathrm{N}_{B}$. Rovelli says then that for such a case " $\mathrm{A}$ and $\mathrm{B}$ have information about one another", and that this information is given by $\mathrm{S}=\log \left(\mathrm{N}_{A} \times \mathrm{N}_{B}\right)-\log \mathrm{N}_{A B}$. We can see clearly that this conception is also true for what we have said above, about the initial emergence of the universe. And we see also why from the beginning on, we have to deal with systems at all: This is because evolution happens in an organized way, and organization is always systematic and based on information (and the material capacity to actualize). Hence, it is even the title of Rovelli's contribution which points into the direction of what we would like to aim at here.

If, on the other hand, we start from our preliminary definition of systems as given in [1], we note the relevance of the definition of interactions for what we are talking about. The definition is: "We call system a network of interacting agents producing a space with a well-defined boundary that is open in the sense of thermodynamics" [4]. Obviously, different from the tradition of talking about interactions in physics so far, what we have to do is to visualize interactions more closely in terms of constraints and relative information transport. Hence, we have not yet achieved the original objective: namely to define matter and information properly, demonstrate their constitutive role as to systems, and represent the results in terms of a unified theory with a closed methodology. This remains to be done in more detail. One step forward could be to attempt a definition for the concept of information based on a similar proposition to the one which is offered by Noether's theorem in the case of energy. However, a first condition for something like this would be a clarification of the question whether entropy-structure would be conserved after all, while entropy alone is not [5]. The ongoing work is being continued. 
The author would like to thank all members of the recently formed study group on the problems mentioned here: José M. Díaz Nafría (León), Annette Grathoff (Vienna), Wolfgang Hofkirchner (Vienna), Muraad Nofal (Munich), Renate Quehenberg (Vienna), Klaus Ruthenberg (Coburg), Tomáš Sigmund (Prague), Verena Streitenberg (Berlin), Doris Zeilinger (Nuremberg).

\section{References}

1. R.E. Zimmermann, Metaphysics of Emergence. Part 1: On the Foundations of Systems (Xenomoi, Berlin, 2015)

2. R.E. Zimmermann, Nothingness as Ground and Nothing but Ground. Schelling's Philosophy of Nature Revisited (Xenomoi, Berlin, 2014)

3. C. Rovelli, Meaning = Information + Evolution, arXiv:1611.02420 [physics.hist-ph]

4. [1], p. 27

5. R.E. Zimmermann: Otherland Revisited. Philosophical Implications of Artificial Worlds, in R.E. Zimmermann, V.G. Budanov (eds.), Towards Otherland. Languages of Science and Languages Beyond (INTAS Vol. 3) (Kassel University Press, 2005), pp. 29-44

Open Access This is an Open Access article distributed under the terms of the Creative Commons Attribution License (http://creativecommons.org/licenses/by/4.0), which permits unrestricted use, distribution, and reproduction in any medium, provided the original work is properly cited. 